\title{
A correlation between fracture toughness and cohesion strength of molybdenum thermal sprayed coatings
}

\author{
Alexandru Paraschiv ${ }^{1, *}$, Gheorghe Matache ${ }^{1}$, Cristian Puscasu ${ }^{1}$ and Mihaela Grigorescu ${ }^{1}$ \\ ${ }^{1}$ Romanian Research and Development Institute for Gas Turbines COMOTI, 220D Iuliu Maniu Ave., \\ Bucharest 6, Romania
}

\begin{abstract}
The Molydenum thermal sprayed coatings on steel substrate using electric arc, atmospheric plasma spray and high velocity oxy-fuel spray were investigated. The tensile bond strength tests were made in order to examine the degree of adhesion/cohesion strength of coatings. The Vickers indentation fracture toughness tests were made on parallel and perpendicular direction to the coating-substrate interface in order to examine the fracture toughness of the material in relationship with the microstructure, indentation direction, hardness and the length of microcracks developed under different loads. It was found that the fracture toughness is influenced by the direction and load of indentation. It was established a correlation between the fracture toughness and cohesion strength of Mo coatings and a general trend was described
\end{abstract}

\section{Introduction}

The Molybdenum thermal spray coatings are used for many applications in sectors of the transport industry, such as automotive and aerospace industries. These are used to increase the corrosion resistance, friction control and thermal insulation to substrate and in particular, for wear resistance [1]. The molybdenum coatings can be also used in railway industry for dimension reconstruction and protection against abrasive, adhesive, surface fatigue, fretting or corrosion of axles of train [2].

Two critical aspects which affect the quality and lifetime of a coating-substrate system are the propagation behavior of the microcracks and the bonding strength (cohesion/adhesion strength) of the coatings. Firstly, the microcracks in these inhomogeneous thermal coatings are propagating in an unpredictable way and finally will lead to a delamination of the coating. The morphology and propagation behavior of microcraks in thermal sprayed coatings, which can occur when mechanical stresses are applied or as a result of residual stresses generated by the cooling and solidifications of hot Mo splats, define a mechanical property of coatings, called critical stress intensity factor or fracture toughness that is generally used for predicting of the mechanical behavior of brittle materials [3]. The fracture toughness is the ability of the coating to withstand at the highest level of stress intensity without fracturing and can be evaluated by using several methods

*Corresponding author: alexandru.paraschiv@,comoti.ro 
such as scratch testing, double cantilever beams loaded in tension, Chevron notch bar and single-edge notched beams tested in three or four point bending [3-4]. One of the most used methods in recent years, providing good results for the evaluating of fracture toughness of thermal sprayed coatings, is the indentation method by Vickers indenter [3-12]. Beside the accessibility of this method, the major advantage is that the fracture toughness of coatings can be tested locally, on several levels such as, at the coating-substrate interface, also called interfacial fracture toughness, on the perpendicular and parallel direction to the coatingsubstrate interface, between layers or individual splats. The general approach is by correlating the length of microcracks initiated in the corners of the Vickers impressions with different constants and mechanical characteristics of the material, such as hardness and modulus of elasticity.

On the other hand, the functionality of a thermal sprayed coating/substrate system depends on the cohesion strength of the layers and lamellar splats and also the adhesive strength of the coating on its substrate. These two types of bonding strength of thermal sprayed coatings can be identified after a visual or microscopic examination of the failure of coatings tested by tensile bond strength tests, according to ASTM Standard C633. Beside the disadvantages of this method in terms of costs and time for preparation of specimens (machining, gluing, curing, etc.), the penetration of the bonding agent into the coating due to the porosity of the coating, modifying its physical properties, and the impossibility to measure the adhesion strength of the coating when the rupture is cohesive and vice versa, represent the main limitations of this method [5-6]. Apart of this, more than eighty methods are reported to measure the coating adhesion, but many of them are also energy consuming in terms of time, cost and equipment [5]. Therefore, an additional method that is increasingly used for evaluating the adhesion strength due to its simplicity, accuracy and low costs is the Vickers indentation testing at the coating-substrate interface, with results that can be expressed either as a stress intensity factor, with units of $\mathrm{MN} \cdot \mathrm{m}^{3 / 2}$ or as fracture surface energy, with unit of $\mathrm{J} \cdot \mathrm{m}^{-2}$ [5-6]. In the same vein, in the case when the rupture of thermal sprayed coatings is produced only within the layers and splats, the Vickers indentation testing can be used as an additional method for evaluating the cohesion strength by locally testing the coating into splats and between layers.

Based on the propagation behavior of the microcracks developed under the Vickers indentation tests and the fracture modes of the coatings tested by tensile bond strength tests, it can be made an association between two of the most important mechanical properties of thermal sprayed coatings such as, the fracture toughness and cohesion bond strength.

So, the basic aim of this paper is to evaluate and correlate the fracture toughness by indentation tests on parallel and perpendicular to the coating-substrate interface with cohesive strength by tensile bond strength tests of molybdenum coatings obtained by three thermal spray methods, electric arc (EA), atmospheric plasma spray (APS) and high velocity oxygen fuel (HVOF).

\section{Materials and procedures}

The support material of the coating-substrate system is an A5-T steel that is currently used for the railway axle manufacturing. This steel support was coated with high purity $(99.9 \%)$ Mo by electric arc (EA), atmospheric plasma spray (APS) and high velocity oxy-fuel spray (HVOF). For EA method it was used an AWS 400 Arc Spray System using Mo wire of 1.6 mm diameter (Sulzer Metco 8228), and for APS and HVOF method it were used a plasma spray torch (Metco 7M with F4 gun) and a HVOF powder spray system (MachJet 2700) respectively, using Mo powder with nominal particle size distribution of $-75+25 \mu \mathrm{m}$ (Metco $63 \mathrm{NS}$ ). For the evaluating of the bonding strength of these coatings it were used two cylindrical specimens of $\varnothing 25,4 \times 25,4 \mathrm{~mm}$ for each type of coating with a coated frontal 
face that was machined until a thicknesses of around $350 \mu \mathrm{m}$ for coatings deposited by APS and EA method and $150 \mu \mathrm{m}$ for coatings deposited by HVOF method. The finished surfaces had a surface roughness Ra of around $2 \mu \mathrm{m}$ for each type of coating and were measured by using a surface roughness tester MarSurf PS10.

Generally, for the evaluating of the adhesion strength of thermal sprayed coatings, the surface of coatings is better to be as-received, with a rougher surface for a good mechanical anchoring between the coating and glue, but in our case the coatings surface was machined because in the real work conditions of the component parts that will be protected by these coatings the thickness and surface roughness are critical technical specifications.

The cylindrical parts with coated surfaces (bond cap) and reference samples with uncoated surface (for testing just the bonding strength of bonding agent) were joined with the cylindrical counter parts (mating cap) using an adhesive agent HTK Ultra Bond 100®, were fixed by using loading weights on a self-aligning device (Fig.1) and were cured at elevated temperature of $150^{\circ} \mathrm{C}$ for 80 minutes, according to ASTM C633 (Standard Test Method for Adhesion or Cohesion Strength of Thermal Spray Coatings, 2008). The testing specimens presented in Fig. 1a and schematically shown in Fig. 1b were loaded along their longitudinal axis with a displacement rate of $0.75 \mathrm{~mm} / \mathrm{min}$ by using the Universal mechanical testing machine INSTRON 8802. After the tensile bond strength tests, the rupture surfaces of coated specimens were visual and microscopic inspected to establish the fracture mode. Based on the specifications of ASTM C633, the adhesion strength of the coating is given if failure is entirely at the coating-substrate interface and also the cohesion strength if rupture is only within the coating layers. For the Mo coatings investigated in this paper, the rupture was cohesive (Fig. 1b) and the cohesion strength values were calculated by dividing the ultimate force by the cross-sectional area of the cylindrical specimens.

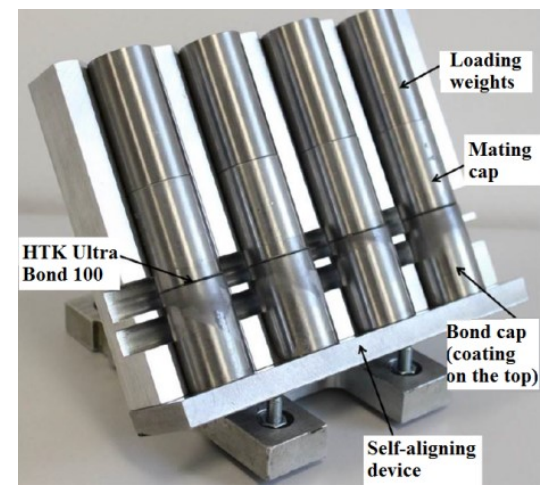

a)

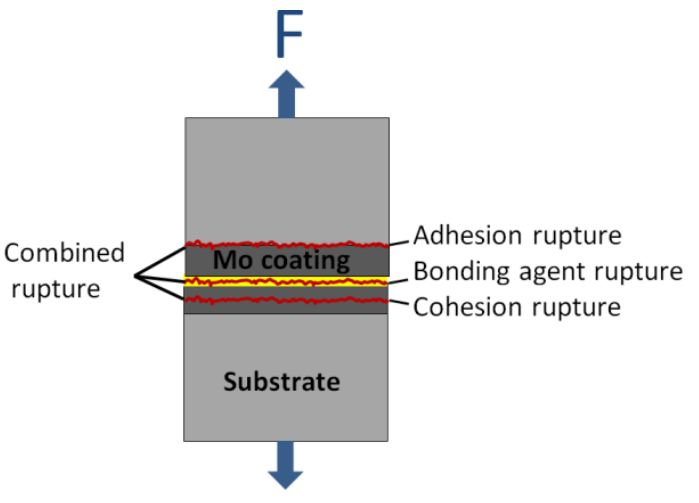

b)

Fig. 1. The preparation of the cylindrical tensile specimens by glueing the coating to the face of a loading fixtures (a) and schematic presentation of different fracture modes generally found in tensile bond strength tests of thermal spray coatings (b)

For the evaluating of the microcracks fracture behavior by calculating the fracture toughness of these anisotropic thermal coatings, we have proposed to test the Mo coatings, on the cross section (perpendicular to the coating-substrate interface or between splats) and on the surface (parallel to the coating-substrate interface or into splats) (Fig. 2a). An average of the local fracture toughness values obtained on both parallel and perpendicular direction to the coating-substrate interface were made, in order to correlate the fracture toughness results with the results obtained by the tensile bond strength tests. For the Vickers indentation tests three steel support samples with $30 \mathrm{~mm}$ in diameter and $10 \mathrm{~mm}$ height were coated on a face by each thermal method with a thickness of around $400 \mu \mathrm{m}$ for EA and APS methods and $150 \mu \mathrm{m}$ for HVOF, were cut in half parts and 
metallographically prepared, according to the indentation direction. By Vickers indentation method were made five measurements of micro and macroindentation for each of the twelve selected loads ranging from 0.098 to $98.1 \mathrm{~N}$. Vickers microindentation hardness tests were performed with a Wilson Hardness ${ }^{\mathrm{TM}}$ 402MVD tester for loads ranging from 0.098 to $9.8 \mathrm{~N}$ (10 to $1000 \mathrm{gf}$ ), while Vickers macroindentation hardness test were performed with an EMCO TEST M4C/R for loads ranging between 19.6 to $98.1 \mathrm{~N}$ (2 to 10 kgf), according to ASTM E384 [7]. The lengths of microcracks, which were developed near the impression of indentation, were measured by using SEM images (FEI Inspect F50) and SEM image analysis software (Scandium) (Fig.4). It was observed that the microcraks generated by Vickers indentation occur after a critical load has been reached and depending on the type of coating and indentation direction they usually propagate on the directions of the indent diagonals. This mechanism of propagation of microcracks appears usually in homogeneous materials and in particular, in brittle materials [3], [8], [9]. Generally, after the Vickers indentation two types of microcracks can be generated, described as Half-penny cracks (median/radial cracks) or Palmqvist cracks (radial cracks) (Fig.2b), where $l$ is the average length of the cracks, $a$ is the half average length of the diagonal and $c$ is the sum of $l$ and $a$.

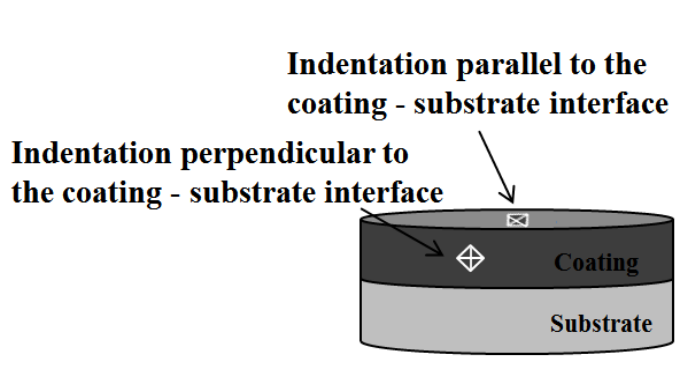

a)

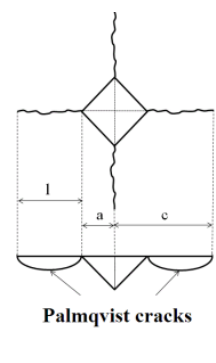

b)

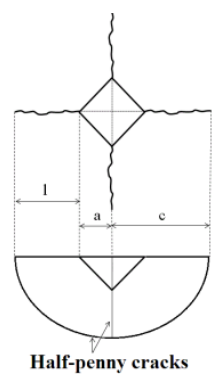

Fig. 2. Schematic illustration of the indentation directions (a) and Palmqvist and interfacial cracks system (b)

To distinguish between these two types of cracks, it can be applied a criterion based on the ratio of cracks length/diagonal indentation [9]. A general rule admits that if $\mathrm{c} / \mathrm{a} \geq 2$, the model is the type Half-penny and if $\mathrm{c} / \mathrm{a}<2$, the model is Palmqvist [3]. Other authors assert that the cracks are Palmqvist type only if $0.25 \leq 1 / \mathrm{a} \geq 2.5$ [10] and they are Half-penny only if $\mathrm{c} / \mathrm{a} \geq 2.5$ [11]. In the case of Mo thermal coatings the average measurements of diagonals and microcracks initiated on the parallel and perpendicular coating-substrate interface indicate that all the microcracks are the Palmqvist type by complying with criteria listed above. The fracture toughness Kic both in parallel and perpendicular to the coatingsubstrate interface was evaluated by using an equation (1) established by Brantner et al. [12] for flame sprayed molybdenum coatings, where $\mathrm{k}$ is a coefficient which depends on the geometry of the used indenter and the tested material, and $\mathrm{P}$ is the load $(\mathrm{N})$.

$$
K_{I C}=k \cdot\left(\frac{P}{a \cdot l^{1 / 2}}\right)
$$

\section{Results and Discussions}

The experimental results of the tensile bonding tests indicate that the Mo coatings deposited by HVOF (HV) method had the highest value of bonding strength (43.3 MPa), followed by APS method $(26.7 \mathrm{MPa})$ and by EA method $(19.2 \mathrm{MPa})$. These differences can be explained by the higher temperature and velocity of sprayed particles which impact the 
substrate and generate a higher level of compressive residual stresses in coatings creating a stronger bond between the layers. Another factor that influences the bonding strength is the dimensions of the Mo splats, which in the case of EA-sprayed coatings are approximately $55 \mu \mathrm{m}$ and $37 \mu \mathrm{m}$ for APS [13] that generates a lower porosity with decreasing of the splats dimension and a higher total contact surface.

The microfractographic investigations of the rupture surfaces for all tested specimens have shown that the failure is produced within the coating layers (Fig. 3b) and individual splats (Fig. 3c), with small adhesive parts removed (no more 2\% of total area) (Fig. 3a) and without a rupture within the glue, which means that the bonding strength calculated is actually the cohesion strength of Mo coatings and it is lower than the adhesion and the glue strength.

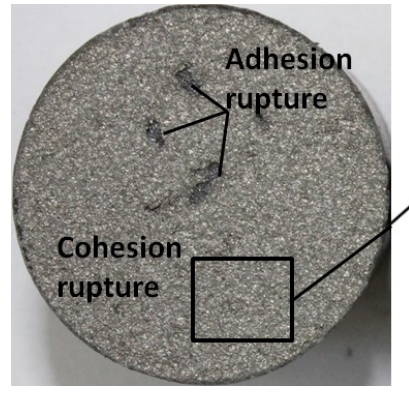

a)

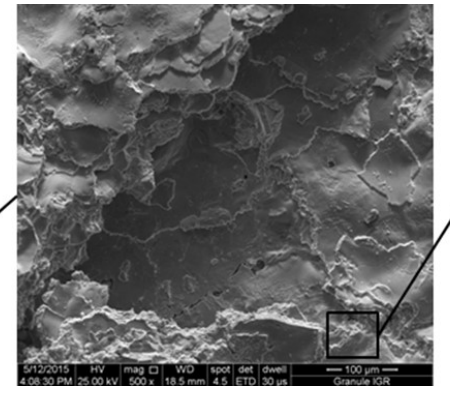

b)

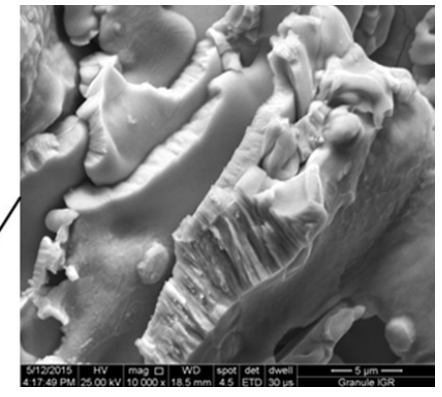

c)

Fig. 3. The general aspect of the coated surfaces after tensile bond strength tests (a) with details of cohesion rupture of Mo layers (b) and fracture of Mo splats (c)

The Vickers indentation method was used for evaluating the propagation behavior of the microcracks and also used as an alternative method for evaluating the cohesion strength following the known rule of interfacial fracture toughness and adhesion strength of coatingsubstrate systems. The Vickers indentations within the specified loads range were performed on parallel (into splats) and perpendicular (between splats) direction to the coating-substrate interface to initiate and propagate microcracks in splats and between layers of coating. In most investigated indentation impressions, two main microcracks grew in two of the four corners of the indentation on both parallel and perpendicular directions (Fig. 4a-b).

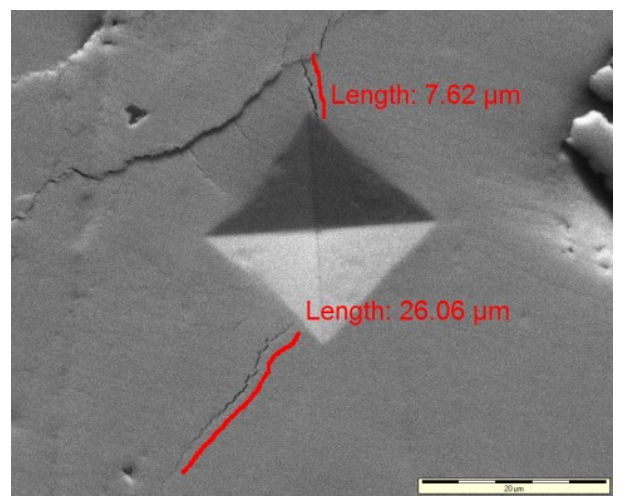

a)

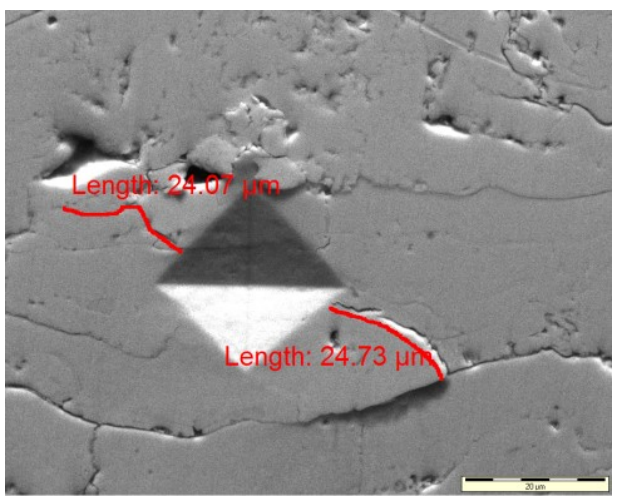

b)

Fig. 4. Vickers indentations on parallel (a) and perpendicular (b) to the coating-substrate interface

It was observed a minimum load (or lower critical load) at which the first microcrack occured and a maximum critical load at which the delamination apperead (e.g. HV2 coating 
after indentation load of $4.9 \mathrm{~N}$, Tabel 1) which depending on the type of coating, indentation direction and thickness of coating. The main limitation of this method was the thickness of coating which has to be higher than $150 \mu \mathrm{m}$ to avoid the delamination. Beside the indentation loads and mechanical properties of coatings, the pre-existing cracks, diameter and thickness of the splats and interface splats play a critical role in propagation of microcracks in a such way that in some cases the cracks propagate randomly (not liniar) and for loads higher than $9.8 \mathrm{~N}$ small secondary microcraks develop near the impression of indentation. All the microcracks that were developed under different indentation loads were of the Palmqvist type and based on their lengths, material hardness and the $k$ parameter according to the indentation direction, it was determined the fracture toughness of the Mo coatings in both parallel an perpendicular direction to the coating-substrate interface.

Table 1 summarizes the fracture toughness values on the parallel (EA1, AP1 and HV1 samples) and perpendicular direction (EA2, AP2, HV2 samples) to the coating-substrate interface for Mo coatings samples deposited by EA (EA1, EA2), APS (AP1, AP2) and HVOF methods (HV1, HV2).

Table 1. Fracture toughness of Mo coatings deposited by APS, EA and HVOF

\begin{tabular}{|l|l|l|l|l|l|l|l|l|l|l|l|}
\hline F[N] & 0.1 & 0.24 & 0.49 & 0.98 & 1.96 & 4.9 & 9.8 & 19 & 29.4 & 49 & 98 \\
\hline EA1 & $*$ & 0.28 & 0.33 & 0.33 & 0.34 & 0.41 & 0.49 & 0.52 & 0.53 & 0.66 & 0.76 \\
\hline EA2 & 0.2 & 0.27 & 0.28 & 0.30 & 0.32 & 0.39 & 0.43 & 0.46 & 0.48 & 0.51 & 0.58 \\
\hline APS1 & $*$ & $*$ & 0.35 & 0.38 & 0.43 & 0.51 & 0.57 & 0.81 & 0.90 & 1.02 & 1.17 \\
\hline APS2 & $*$ & 0.36 & 0.43 & 0.51 & 0.55 & 0.57 & 0.60 & 0.69 & 0.73 & 0.74 & 0.76 \\
\hline HV1 & $*$ & 0.70 & 0.85 & 0.88 & 1.00 & 1.15 & 1.40 & 1.59 & 1.81 & 2.05 & 2.27 \\
\hline HV2 & $*$ & 0.45 & 0.55 & 0.59 & 0.63 & 0.69 & $\sim$ & $\sim$ & $\sim$ & $\sim$ & $\sim$ \\
\hline
\end{tabular}

* no initiated microcraks

$\sim$ delamination appeared

As it can be seen in Table 1, there were significantly differences between the fracture toughness values obtained on these three types of Mo coating, but in all cases the values obtained on the parallel direction were higher than those on perpendicular direction to the coating-substrate interface. This is due to the fact that on parallel direction the most indentations are in the homogeneous splats while on the cross section (or perpendicular to the coating-substrate interface) the indentations are mostly on the interface between the splats which represents the weakest link in a thermal spray coating. Unlike the homogeneous materials for which the fracture toughness is relatively constant irrespective of the indentation load [8], for inhomogeneous coatings, containing pores, oxides, boundaries of splats or containing pre-existing microcracks, the tendency of fracture toughness is to increase with the increase of the indentation loads. This tendency can be explained by the deformation field which is not self similar for different indentation and thus leads to microcrack driving stress field that varies with indentation load [9] or by the R-curve effect of the toughness [12]. Two other assumptions can explain this local fracture behavior of thermal sprayed Mo coatings. The first assumption is that by increasing indentation loads the microcracks propagate towards the boundaries of splats, pores or the pre-existing microcracks. In such case, it is encountered an increase of indentation diagonals length by increasing the indentation load inversely proportional to the length of the developed microcracks. As a result, a higher value of fracture toughness is calculated. The second assumption can be taken into consideration, in particular for indentation loads

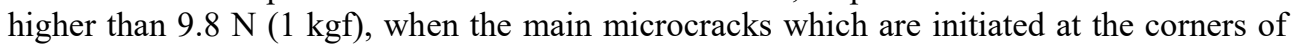
Vickers indentation branch into smaller secondary microcracks. Therefore, the inhomogeneity of Mo coating can prevent the increase in length of microcracks and favors the increasing of fracture toughness values on both indentation directions. The propagation 
behavior of the microcracks in thermal sprayed coatings in terms of fracture toughness indicates that the interface between splats is the weakest link in a thermal spray coating. This aspect is also confirmed by the tensile bond strength tests due the major fracture mode within the Mo layers. Between these two mechanical properties it can be made an association in terms of resistance to crack propagation because before the failure of a thermal sprayed coating, the microcracks appear, grow and propagate through the layers of coatings and finally, will contribute to a cohesive fracture, in the case of tensile bond strength testing, or to a delamination, in the case of indentation testing.

However, similar associations between results from the tensile adhesive tests and interfacial indentation tests were made for thermal sprayed coatings and it was concluded that the interfacial fracture toughness is a suitable parameter to characterize the adhesion of thermal sprayed coatings [5] [14]. To correlate the local fracture toughness with the cohesion strength of Mo coatings investigated, it was considered an average fracture toughness of the values obtained for each thermal spray method $(0.419 \mathrm{Mpa} \cdot \sqrt{\mathrm{m}}$ for EA, $0.623 \mathrm{Mpa} \cdot \sqrt{\mathrm{m}}$ for APS and $1.081 \mathrm{Mpa} \cdot \sqrt{\mathrm{m}}$ for HVOF). Therefore, in Fig. 5 it is presented a correlation between the fracture toughness Kic and cohesion strength of Mo coatings deposited by EA, APS and HVOF methods. The linearity of the cohesion strength as a function of fracture toughness can be expressed as the slope of the line that describes the increasing of cohesive strength with increasing of fracture toughness.

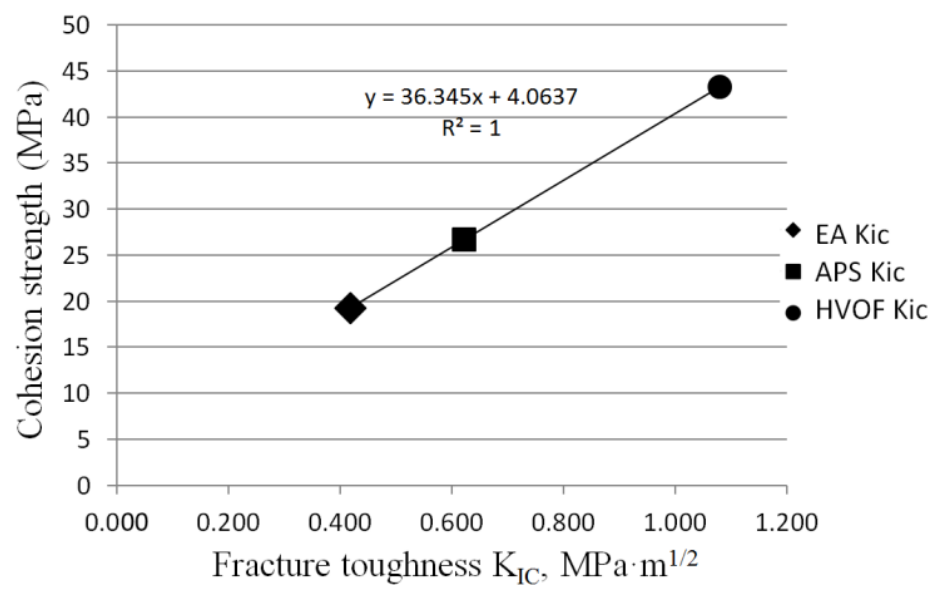

Fig. 5. Correlation between fracture toughness Kic and cohesion strength

This trend shows a superior resistance to crack propagation of Mo coatings deposited by HVOF and consequently, a higher cohesion strength value than those of the coatings deposited by APS and EA methods. The results obtained by indentation tests for evaluating the fracture toughness of Mo coatings are in the range $0.419-1.081 \mathrm{MPa} \cdot \mathrm{m}^{1 / 2}$, which is in good agreement with the values obtained by Brantner et al. for flame sprayed molybdenum coatings [12], but the results obtained by tensile bond strength tests for similar coatings and testing conditions are very scattered. For example, for Mo coatings deposited by plasma spraying with a thickness between $300-350 \mu \mathrm{m}$, it was calculated an adhesion strength values of 33-35 $\mathrm{MPa}$ [15], a value of around $52 \mathrm{MPa}$ for Mo coatings with a thickness of around $400 \mu \mathrm{m}$ [16] or values between 18-52 $\mathrm{MPa}$ in another case [17]. Also, for Mo coatings deposited by high velocity combustion wire spray process (similar to the HVOF process) it was reported a cohesive failure of Mo coatings and tensile bond strength values between 19.7-27.9 MPa [18]. Beside the influence of thickness, which is well know that influence the bonding strength of thermal sprayed coatings, these differences can originate due to many causes. Firstly, the thermal spray equipment, spray distance parameters, raw 
material for spraying and substrate preparation can differ from case to case and generate these different performances of bonding strength. Other factors which can influence the bonding strength values are the bonding agent that can cause an overestimate of the adhesion strength due penetration into the coating towards the interface [5], and also the state and the roughness of the surface. In our case, the coated surfaces were machined and a surface roughness value $\mathrm{Ra}$ of around $2 \mu \mathrm{m}$ was obtained for all tested coatings, because in the real work conditions of the component parts coated by Mo coatings, such as shafts and axles, this is the critical technical specification. During the tensile bond strength tests such a smooth coating surface had a critical role for the mode of rupture and bonding strength performance because it did not allow a good mechanical anchoring between surface coated and bonding agent and just a few superior layers of coating experienced rupture by delamination. Also, internal tension stresses were induced in coating during machining of surface that weakened the link between Mo layers, in particular on the superior part of the coating-substrate system where a partial rupture of the coating (cohesion rupture) was generated and not a total rupture (adhesion rupture).

Therefore, as the relationship between the interfacial fracture toughness and adhesion strength can be employed to evaluate and anticipate the mechanical properties of coatings in terms of fracture behavior and bonding strength, the general trend which has been found in this paper for the relation between the fracture toughness and cohesion strength can be used in the cases when the rupture of coatings is produced within the layers, in particular for thermal sprayed coatings where the interface splats is the weakest link in the coating.

\section{Conclusions}

Based on the experimental results obtained in this study, general conclusion can be drawn.

Mo coatings deposited by three thermal spray techniques such as EA, APS and HVOF methods are investigated by tensile bond strength and Vickers indentation tests in order to evaluate and correlate the two of most important mechanical properties of the thermal sprayed coatings such as, cohesion strength and fracture toughness.

The micro fractographic investigations of the rupture surfaces of specimens tested at tensile bond strength tests, indicated that the failure of all coatings is mostly produced within the layers and individual splats and therefore, the cohesion strength was calculated according to ASTM Standard C633. It was obtained a mean value of cohesion strength of 43.3 MPa for HVOF-sprayed coatings, 26.7 MPa for APS-sprayed coatings and 19.2 $\mathrm{MPa}$ for EA-sprayed coatings.

The Vickers indentations of Mo coatings on both parallel and perpendicular direction to the coating-substrate interface generate Palmqvist cracks at the corners of impressions under different indentation loads and depending on the Mo coating type and the indentation direction. The fracture toughness as a function of microcracks size and indentation load of Mo coating obtained by HVOF method is higher than the fracture toughness of Mo coatings obtained by APS and EA methods, under each indentation load, and indentation directions due the splats dimensions, porosity, oxides, boundaries splats or pre-existing microcracks.

It was shown that between these two mechanical properties, cohesion strength and fracture toughness, it can be made a similar correlation that it usually is made between adhesive strength and interfacial fracture toughness of thermal sprayed coatings. The general trend is expressed as the slope of the line that describes the tendency of fracture toughness to increase with the increase of cohesive strength and it was concluded that the fracture toughness is a suitable parameter to characterize the cohesion strength of thermal sprayed coatings. 
This work has been done within Partnerships in Priority Areas Program - PN II, implemented with the support of MEN - UEFISCDI, project no. 81/2014.

\section{References}

1. J. Shields, Applications of Molybdenum Metal and its Alloys, IMOA (2013)

2. F. S. M. Kasparova, 18.-20.5 Brno Metals 2011, Czech Republic (2011)

3. A. Moradkhani, H. Baharvandi, M. Tajdari, H. Latifi, J. Martikainen, J. Adv. Ceram. 2, 1 (2013)

4. Z. Xia, W.A. Curtin, B.W. Sheldon, Acta Materi. 52, 3507-3517 (2004)

5. G. Marot, J. Lesage, Ph. Démarécaux, M. Hadad, St. Siegmann, M.H. Staia, Surf. Coat. Technol. 201, 2080-2085 (2006)

6. Y. Yamazaki, M. Arai, Y. Miyashita, H. Waki, and M. Suzuki, JTTEE5 22, 1358-1365 (2013)

7. ASTM Standard ASTM E384-10e2. Standard Test Method for Knoop and Vickers Hardness of Materials, ASTM International (2010)

8. R. Spiegler, S. Schmauder, L.S.Sig., J. Hard. Mater. 1, 147-158 (1990)

9. T. Lube, J. Eur. Ceram. Soc. 21, 211-218 (2001)

10. E. E. Medeiros, A.M.S. Dias., IJRRAS 17, 9-18 (2013)

11. K. K. Bamzai, P. N. Kotru, B. M Wanklyn, J. Mater. Sci. Technol. 16, 405-410 (2000)

12. H. P. Brantner, R. Pippan and W. Prantl., J. Therm. Spray. Techn. 12, 560-571 (2003)

13. G. Matache, C.Puscasu, A. Paraschiv and O.Trusca, AMM 811, 19-23 (2015)

14. A. Kishi, S. Kuroda, T. Inoue, T. Fukushima, and H.Yumoto, JTTEE5 17, 228-233 (2008)

15. J. Ružbarský, A. Panda, SpringerBriefs Appl. Sci. Technol. (1st ed. 2017) (2016)

16. A. Vencl, S. Arostegui, G. Favaro, F. Zivic, M. Mrdak, S. Mitrovic, V. Popovic. Tribol. Int. 4, 1281-1288 (2011)

17. M. Mrdak, A. Vencl, M. Ćosić, FME Trans. 37, 27-32 (2009)

18. S.C. Modi \& A. Calla, J. Therm. Spray. Techn. 10, 480-486 (2001) 